Saudi Journal of Oral and Dental Research

Abbreviated Key Title: Saudi J Oral Dent Res

ISSN 2518-1300 (Print) |ISSN 2518-1297 (Online)

Scholars Middle East Publishers, Dubai, United Arab Emirates

Journal homepage: https://saudijournals.com

Case Report

\title{
Rare Occurrence of Permanent Impacted First Molars: A Series of 2 Case Reports
}

Afaf Ali M Hakami ${ }^{1 *}$, Yasser Mohammed Essa Masmali², Shaker Yasir Alsulimani ${ }^{3}$, Salehah Ahmed Ali Jerah ${ }^{4}$, Fareedi Mukram Ali $^{5}$

${ }^{1}$ General Dental Practitioner, Ministry of Health, Jazan, KSA

${ }^{2}$ Dental Intern, College of Dentistry, Jazan University, Al Maarefah Rd, Jazan Saudi Arabia

${ }^{3}$ Dental Intern, Al Farbi Dental College, Jeddah, KSA

${ }^{4}$ General Dental Practitioner, Jazan, KSA

${ }^{5}$ Assistant Professor, College of Dentistry, Jazan University, Al Maarefah Rd, Jazan Saudi Arabia

DOI: $10.36348 /$ sjodr.2021.v06i01.009

| Received: 01.01.2021 | Accepted: 09.01.2021 | Published: 14.01.2021

*Corresponding author: Dr. Afaf Ali M Hakami

\section{Abstract}

In human dentition, permanent tooth impaction is relatively common. But, the first permanent molars are rarely impacted with a reported prevalence of $0.02 \%$ for permanent maxillary first molar and fewer than $0.01 \%$ for the permanent mandibular first molars. The etiology for the failure of eruption can be variable. It is usually an symptomless pathology. Diagnosis in early stage and treatment of impacted permanent molars contributes to most favourable outcomes and good long-standing prognosis by lessening of complication. Here we are presenting a case reports of 2 cases of permanent first impacted molars.

Keywords: Impacted teeth, First molar, Permanent teeth.

Copyright () 2021 The Author(s): This is an open-access article distributed under the terms of the Creative Commons Attribution 4.0 International License (CC BY-NC 4.0) which permits unrestricted use, distribution, and reproduction in any medium for non-commercial use provided the original author and source are credited.

\section{INTRODUCTION}

The permanent teeth impaction is generally a frequent incidence. The permanent teeth impaction usually occurs in the downward order of third molars, maxillary cuspids, mandibular premolars, mandibular canine, maxillary premolars, maxillary central incisors, and mandibular second molars. But the first permanent molars are rarely impacted [1].

Tooth retention has been recognized to an alteration of the dental follicle, which is unable to initiate the metabolic processes leading to resorption of bone and eruption [2].

The aetiological factors accountable for impaction of tooth can be classified in two main groups. First, local factors, like an bone deposition increase, disturbances of the primary dentition, trauma, supernumerary malocclusion or malposition of adjacent teeth, long-standing retention of primary teeth, and local pathosis [3].
A second group consists of systemic factors, including childhood diseases, hereditary factors, ankylosis of the TMJ, cleft palate, syphilis, and cleidocranial dysostosis [3].

In this paper, we are describing the case reports of permanent first impacted molars.

\section{CASE REPORT}

Case 1

A 31 year old male patient came to the dental hospital with a complaint of absent tooth in the mandibular left posterior region since childhood. On clinical examination, it was seen that the permanent mandibular first molar was missing and the mandibular second permanent molar of that side was mesially tilted. Patient was not having any systemic history. On radiographical examination, the permanent mandibular first molar was found impacted. It was present near to the lower border of mandible. Also the distal root found to have severe distal curvature. As the patient was not having any complaint regarding impacted tooth, it was kept as it was and missing tooth was replaced (Figure1). 


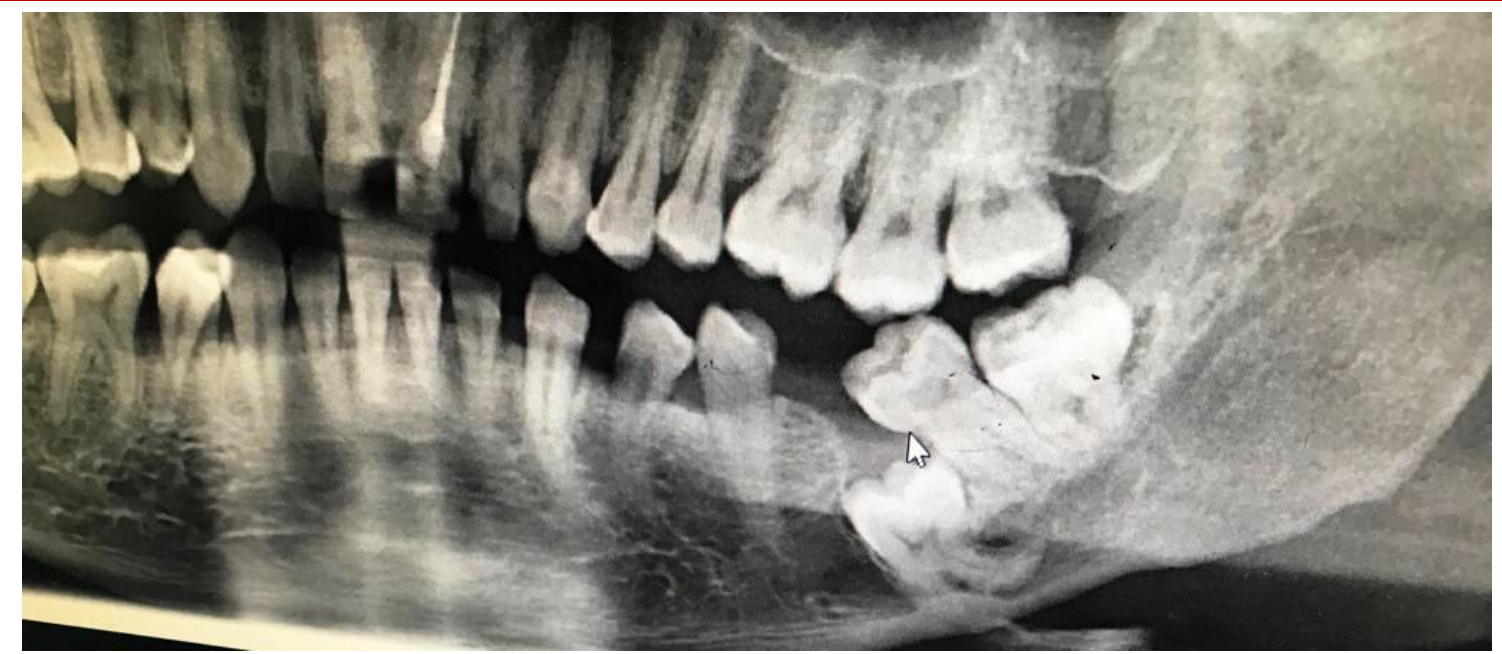

Fig-1: OPG showing impacted mandibular first molar of left side

Case 2

A 38 year old female patient came to the clinic with a complaint of spacing between maxillary right posterior region. On clinical examination, the maxillary right permanent first molar was found missing. Patient was not having any dental or medical history. On radiographical examination, the permanent maxillary first molar of right side was found impacted. The permanent second molar was mesially tilted (Figure-2).

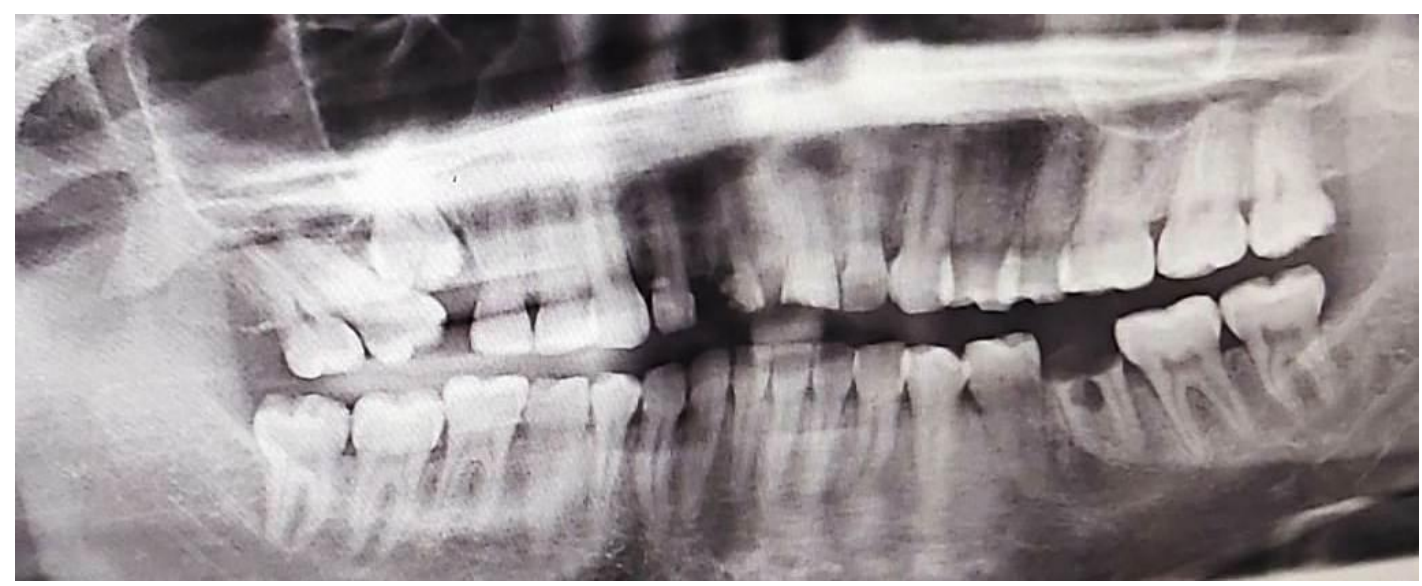

Fig-2: OPG showing impacted maxillary first molar of right side

\section{DISCUSSION}

The teeth which are prevented from erupting by some physical barrier in the path of eruption are called as impacted teeth. If eruption has been prevented but physical barrier or an abnormal position eruption path is absent, then the tooth is called as primarily retained. Secondary retention is the stoppage of tooth eruption after emergence, where the cause is neither a physical barrier in the eruption path nor abnormal positioning [4].

The Diagnosis of impacted permanent molars is frequently based on clinical or regular radiographic examination [5].

The permanent first and second molars play a significant in the growth of dental and facial anatomical structures [6]. It has been reported that impaction of first or second permanent molars leads to retrognathia inferior [7].
It has been reported from the literature that in general the eruption problems of permanent molars occur more commonly in the mandible than in the maxilla. Also the second molar impaction rate is more than first molar [7].

In most of our review of literatures it was found that first permanent molar impactions are due to ectopic eruption and it may cause resorption of distal root of deciduous second molars or even premature exfoliation of the same. In all these conditions generally multiple teeth are concerned [1].

An impacted first molar can cause caries, root resorption and periodontal breakdown of adjacent teeth [8]. 
Based on Winter's classification systems, impacted molars can be classified as vertical, distoangular, mesioangular, or horizontal in position according to tooth angulation. The occlusal plane usually help to check the depth of the impacted molars [9].

The options for the treatment of an impacted molar include extraction, surgical uprighting, transplantation, removal of any possible barrier, surgical-orthodontic approach, and dental implant replacement [10].

Orthodontic management of teeth should go after in the incident of abnormal tooth positioning, lack of space in the dental arch, malocclusion, or if not expecting spontaneous eruption. Due to the little occurrence of first permanent molars impaction, there is deficient in regularity in the management of impacted teeth, and available reports are chiefly based on case reports and mesially inclined molars [4].

\section{CONCLUSION}

The permanent first molars impaction is an uncommon phenomenon. Early diagnosis of such condition is necessary for the proper management. Clinicians must inform the patient of the potential risks and possible benefits of treatment alternatives before making the final treatment plan.

\section{REFERENCES}

1. Bhagat, B. A., Nagrik, A., \& Yemle, S. B. (2016). Management of Impacted Permanent First Molar A Case Report. IOSR Journal of Dental and Medical Sciences, 15(2):105-107.

2. Babacan, H., Ay, S., \& KÖşger, H. H. (2006). Impacted permanent first molars: two case reports. International dental journal, 56(1), 49-54.
3. Machado, A. W., \& Braga, E. (2014). Unilateral impaction of a maxillary first molar treated in the transitional dentition. Pediatric dentistry, 36(7), 502-504.

4. Bereket, C., Özkan, N., Sener, Ý., Kara, M. I., Aktan, A. M., \& Arici, N. (2011). Retrospective analysis of impacted first and second permanent molars in the Turkish population: A multicenter study. Med Oral Patol Oral Cir Bucal, 16 (7):e874-8.

5. Proffit, W. R. (1978). Equilibrium theory revisited: factors influencing position of the teeth. The Angle Orthodontist, 48(3), 175-186.

6. Keris, E. Y., Bozkaya, S., Öztürk, M., \& Güngör, K. (2016). Prevalence and characteristics of impacted permanent molars in a Turkish subpopulation. Journal of Oral and Maxillofacial Radiology, 4(3), 45-49.

7. Panda, S. (2011). First permanent mandibular molar impaction- a case report. Annals and Essences of Dentistry, 3(2):73-4.

8. Mariano, R. C., de Carvalho Freire Mariano, L., \& de Melo, W. M. (2006). Deep impacted mandibular second molar: a case report. Quintessence International-English Edition-, 37(10), 773-776.

9. Fu, P. S., Wang, J. C., Chen, C. H., Huang, T. K., Tseng, C. H., \& Hung, C. C. (2012). Management of unilaterally deep impacted first, second, and third mandibular molars. The Angle Orthodontist, 82(3), 565-571.

10. Valmaseda-Castellón, E., De-la-Rosa-Gay, C., \& Gay-Escoda, C. (1999). Eruption disturbances of the first and second permanent molars: results of treatment in 43 cases. American journal of orthodontics and dentofacial orthopedics, 116(6), 651-658. 